\title{
The Effects of Steroid Hormones on Adipogenic Markers in 3T3-L1 Adipocytes
}

\author{
G. Alshammari ${ }^{1 *}$, J. Brameld², M. Lomax ${ }^{2}$ \\ 1Department of Food Science and Nutrition, King Saud University, Riyadh, Saudi Arabia. \\ 2Molecular Nutrition Division, College of Bio-Sciences, the University of Nottingham, Nottinghamshire, UK. \\ *Corresponding author. Tel.: +44 0115 9516120; email: sbxga1@nottingham.ac.uk \\ Manuscript submitted May 10, 2014; accepted August 3, 2014. \\ doi: $10.7763 /$ ijbbb.2014.v4.388
}

\begin{abstract}
Obesity is a crucial health issue, being a risk factor for the development of chronic metabolic diseases such as type 2 diabetes, hypertension, hyperlipidemia, cardiovascular diseases, and some types of cancer. Traditional methods to control obesity by decreasing food intake, increasing exercise, and medical interventions have not been successful and more fundamental knowledge at the level of genes is required. In men, low levels of testosterone have emerged as an important medical concern since testosterone deficiency is related to the development central obesity. White fat cells were grown in DMEM supplemented with 10\% FBS. Lipid accumulation in 3T3-L1 adipocytes was assessed by staining for $1 \mathrm{hr}$ in a freshly diluted Oil Red O. RNA was extracted, cDNA synthesised and mRNA quantified by using standard qRTPCR. 3T3-L1 treated with sex steroids $(5,15,30 \mathrm{nM})$ for 13 days exhibited a non-significant decreased in adipogenic markers (Resistin and FABP 4) without clear change in Adipo Q expression. In the conclusion, we found Adipogenic markers were not affected by steroid hormones, and these data demonstrate the need to conduct more results to investigate the effect of sex steroid at different doses and time courses especially during early stage of differentiation on transcription factors such as PPAR gamma 2, CEBP alpha, and CEBP betta.
\end{abstract}

Key words: Steroid hormones, obesity, chronic diseases.

\section{Introduction}

Currently, there are around 1.7 billion people worldwide suffering from obesity and there has been a marked increase in the prevalence of obesity and overweight individuals around the world during the last two decades [1]-[3]. Obesity is a crucial health issue since it is an important risk factor for chronic metabolic diseases, including metabolic syndrome, type 2 diabetes, hypertension, hyperlipidaemia, and cardiovascular diseases. Other chronic diseases such as Alzheimer's, gallstones, kidney stones, obstructive sleep apnea, and some types of cancer, have been also linked to obesity [4], [5]. According to WHO reports, The National Institutes of Health (NIH) and World Health Organization (WHO) have predicted that by 2015, more than 2.3 billion adults in the world will be diagnosed as obese or overweight and more than 700 million people will be obese. As a result, efforts to decrease and control the obesity rate are urgently needed [6]. In addition to its impact on health, obesity also has significant economic consequences. It can lead to a decrease people's productivity due to sick leave and long-term work disability and also has major impact on the cost of healthcare services [7], [8]. 


\section{Causes and Solutions}

Although there have been attempts to create new strategies to treat obesity, the available dietary and pharmacological interventions have a poor success rate. Consequently, new treatments are required to combat obesity [9], [10].

Obesity occurs when dietary intake exceeds energy expenditure for prolonged periods causing triacylglycerol (TAG) storage in white fat cells. Therefore, an understanding of the biology of adipocytes and molecular mechanisms involved in fat storage is necessary to produce new treatments for obesity. However, not all obese individuals suffer from chronic diseases or have the same probability to develop associated diseases. This may be because gender and the distribution of fat in the body play a significant role in determining the risk of developing medical problems (Fig. 1) [4]-[12]. Men tend to accumulate visceral fat (android fat) while women accumulate subcutaneous fat (gynoid) [13]. Males are more likely to develop chronic diseases than females who preferentially store fat in subcutaneous adipocytes.

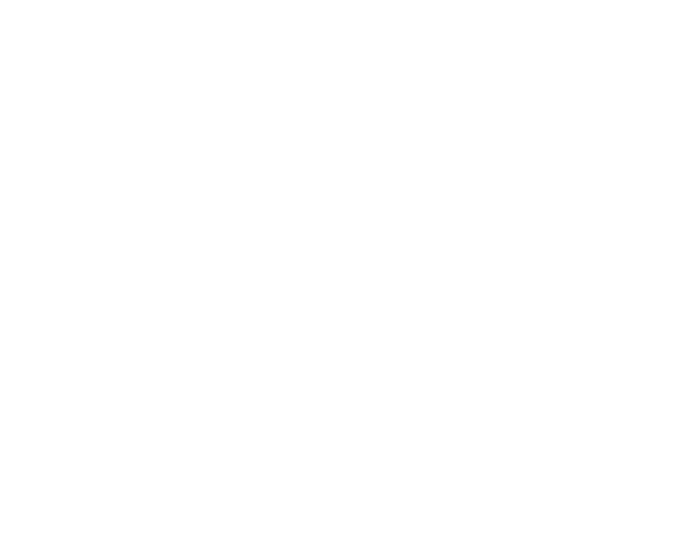

Fig. 1. Distribution of fat and metabolic syndrome.

The mechanism for the gender-dependent accumulation of fat in different regions is not well understood but has been suggested to be related to the effect of sex steroids on increased proliferation of preadipocytes, increased differentiation and increase storage of fat in a mature adipocytes [14]. Many studies have revealed that visceral fat (abdominal) is associated with metabolic disorder whereas subcutaneous fat (peripheral fat distribution) is not involved in these diseases [15] Yamamoto et al. (2010) suggested that visceral but not subcutaneous fat has a strong correlation with cancer [16], [17].

The presence of steroid hormones receptors in adipose tissue indicates that proliferation and differentiation of adipocytes can be regulated by steroid hormones [18]. In men, low levels of testosterone have emerged as an important medical concern since testosterone deficiency is related to the development central obesity and associated cardiovascular diseases, type 2 diabetes and increased mortality and morbidity [19], [20]. There is an age related decline in total and free testosterone levels in men associated with an increased adipose and decreased muscle mass [21].

In this study of our project, we aimed to explain the mechanism by which sex steroids could influence fat cell lipid accumulation and gene expression of adiponic markers, By using a 3T3-L1 mouse preadipocyte cell line. We hypothesized that different doses of androgen and oestrogens lead to decrease fat accumulation in fat cells.

\section{Materials and Methods}

\subsection{Chemical Reagents}

Testosterone, $17 \beta$-estradiol and Dihydrotestosterone (DHT) were purchased from Sigma Company. Other 
cell culture reagents and routine chemicals were supplied either Sigma or Invitrogen.

\subsection{Cell Culture}

3T3-L1 adipocytes were obtained from the American Cell Culture Collection. Cells were cultured in 6-well plates in growth medium (10\%FBS in DMEM; Gibco). The medium was changed every two days and 2 days post-confluent 3T3-L1 preadipocytes were treated with DMEM containing a final concentration of $250 \mathrm{nM}$ dexamethasone, $0.5 \mathrm{mM} \mathrm{3-isobutyl-1-methylxanthine,} \mathrm{and} \mathrm{10 \%} \mathrm{FBS} \mathrm{for} 48 \mathrm{~h}$. The medium was then changed to DMEM containing 10\% FBS and insulin (167 nM) for an additional 15 days. During each treatment control, testosterone, dihydroxytestosterone DHT, and estradiol $(5,15,30 \mathrm{nM})$ were added into three wells and the medium containing was changed every two days. The treatments were added at day 2 . With this protocol, greater than $90 \%$ of adipocytes differentiated.

\subsection{Oil-red O Staining}

To prepare the cell lipid staining solution, $1 \mathrm{~g}$ of oil red 0 (sigma) was added to $100 \mathrm{ml}$ of isopropanol and stored at room temperature and filtered before use. Cells were washed with PBS two times and fixed with formaldehyde $(10 \%)$ for at least $30 \mathrm{~min}$ at room temperature. They were stained with a $0.5 \%(\mathrm{w} / \mathrm{v})$ oil red 0 in isopropyl alcohol/water $(60 / 40, \mathrm{v} / \mathrm{v})$ solution, and washed twice with PBS. The cells were photographed and analysed with Image -Pro +5.1.

\subsection{Analysis of Target Gene Expression by Real Time PCR}

Total RNA was isolated from differentiated 3T3-L1 cells using Trizol (Invitrogen) by following the manufacturer's recommendations. The medium was removed and $1 \mathrm{ml}$ of Trizol was added to each well, the cells were detached by scraping with a plastic spoon and then as soon as possible, transferred into a $1.5 \mathrm{ml}$ tube. The cells were broken down by repeated passage through a 19G needle using a $1 \mathrm{ml}$ syringe and the tubes were frozen at $-80^{\circ} \mathrm{C}$. To extract the RNA, tubes were thawed by incubating at room temperature for 3-5 minutes, and then $200 \mu \mathrm{l}$ chloroform was added and the tube contents were mixed by using a whirly mixer for $15 \mathrm{sec}$. The tubes were then centrifuged at $12000 \mathrm{~g}$ for 10 minutes at $2-4{ }^{\circ} \mathrm{C}$, and the top aqueous layer was carefully transferred into a new tube. $400 \mu \mathrm{l}$ of pure isopropanol was then added and the tubes were incubated at room temperature for $10 \mathrm{~min}$. They were then centrifuged at $12000 \mathrm{~g}$ for $10 \mathrm{~min}$ at $2-8^{\circ} \mathrm{C}$. The supernatant was discarded and the pellet was washed with $1 \mathrm{ml} 75 \%$ ethanol. This was repeated twice and the pellets were dried in air followed by dissolving the RNA in $40 \mathrm{ml}$ of RNAase-free water. RNA was stored at $-80^{\circ} \mathrm{C}$ until cDNA synthesis.

For cDNA synthesis, each pellet was mixed with $5 \mu$ l DNase 10x buffer (promega) and $5 \mu$ l DNase (promega) and then was incubated at $37^{\circ} \mathrm{C}$ for $30 \mathrm{~min} .200 \mu \mathrm{l}$ of isoamylalcohol was added then the tubes were centrifuged at $13000 \mathrm{~g}$ for $5 \mathrm{~min}$. The upper layer containing RNA was transferred into a new tube and then $15 \mu \mathrm{l}$ of $3 \mathrm{M} \mathrm{Na}$ Acetate and $375 \mu \mathrm{l}$ of pure ethanol were added and tubes mixed. All the tubes were centrifuged at $13000 \mathrm{~g}$ for $15 \mathrm{~min}$ at $2-4{ }^{\circ} \mathrm{C}$, the supernatant discarded, and the pellets were washed twice with 75\% ethanol. The pellets were dried for 10 min RNA quality was assessed with a nanodrop machine. Absorbance of $1.5 \mu \mathrm{L}$ from each sample was spectrophotometrically determined at $260 \mathrm{nM}$ and $280 \mathrm{nM}$ against a water blank at $260 \mathrm{nM}$. The ratio of purity of RNA was between the absorbance at 260 and $280 \mathrm{nM}$ ranged from 1.8 to $2.100 \mathrm{ng} / \mu \mathrm{L}$ for each sample was calculated by adding RNase free water. To complete cDNA synthesis, random primers, $\mathrm{H}_{2} \mathrm{O}$ were added and incubated at $70{ }^{\circ} \mathrm{C}$ for $5 \mathrm{~min}$ in a PCR machine. The following mixture Moloney Murine Leukemia Virus Reverse Transcriptase (MMLV) reverse transcriptase buffer, Nucleotides, RNase inhibitor, MMLV reverse transcriptase, and RNase water were added. All of them were mixed and spun down then incubated at room temperature for $10 \mathrm{~min}$ then returned into PCR machine to incubate them at $42{ }^{\circ} \mathrm{C}$ for 60 min. mRNA content of treated cells was measured by RT-PCR. 
cDNA samples were mix with SYBR Green I master (containing HotStar Taq DNA polymerase, QuantiTect SYBR Green PCR Buffer, dNTP mix and SYBR Green I) and mixed with $1.25 \mu \mathrm{l}$ of each primer.

The forward and reverse primers were TGAGGGTCTGGAAATGAA/GGCCAGCCTGGACTATATGA for mouse resistin, TTGACGAAGTCACTGCAGATGA/CAGGACACCCCCATCTAAGGT for FAB4 and AAAGGAGAGCCTGGAGAAGC/AAAGGAGAGCCTGGAGAAGC for Adipo Q. Refference gene is mouse 36B4, forword is TCCAGGCTTTGGGCATCA and its reverse is TTATCAGGTGCACATCACTCAGAAT.

\section{Statistics}

Data are presented as mean \pm sem. Differences between the groups were analyzed by one way ANOVA using GenStat 15.

\section{Results}

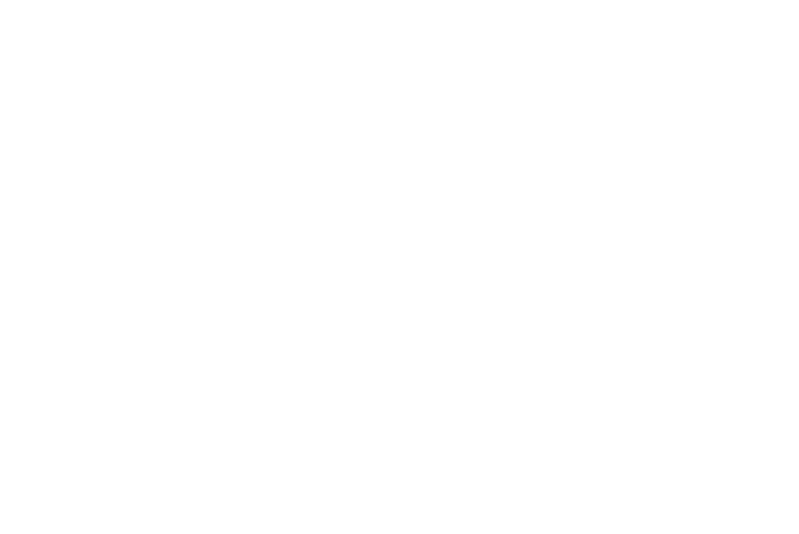

Fig. 2. Testosterone, DHT and Estradiol did not inhibite adipogenic differentiation of 3T3-L1 preadipocytes.

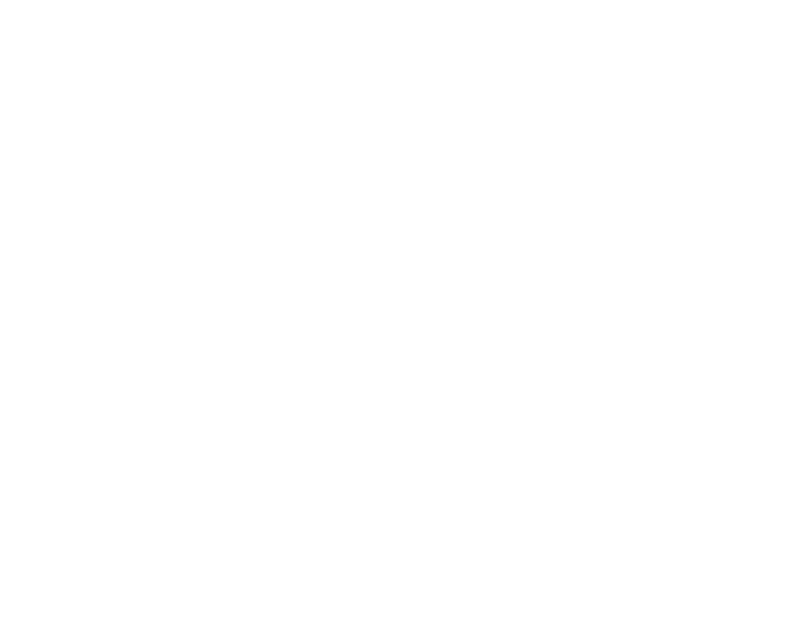

Fig. 3. Effect of steroid hormones added during differentiation on 3T3-L1 differentiation. At day 15, cells were stained with Oil Red 0 to assess lipid accumulation and visualized by light microscope. Values are fold induction relative to the control and expressed as means +/- SEM of three wells.

Some reports have indicated that increased adipocyte during the aging process may contribute to the increase in the incidence and severity of obesity observed in older individuals [22]. 3T3-L1 accumulation of triglyceride droplets, differentiation and adipocyte-specific gene expression were evident in 3T3-L1 cells on the day 13 following $2 \mathrm{~d}$ of MDI. Control or sex steroid treatment, as shown by positive staining with Oil red 0. Treatment of cells with steroid hormones (testosterone, dihydrotetosteron, and $17 \beta$-estradiol) at 
different concentrations did not alter lipid accumulation (Fig. 2) and this was confirmed by quantitative assessment of lipid droplet by using imaging software, differences were considered as statistically significant at $P<0.05$ (Fig. 3).

In 3T3-L1 cells differentiated by using the standard protocol for 15 days, expression of adipogenic markers (Resistin, AdipoQ and FABP4) was not altered by treatment of cells with steroid hormones (testosterone, dihydrotetosterone, and $17 \beta$-estradiol) at different concentrations for the last $24 \mathrm{hrs} \mathrm{(Fig.} \mathrm{4,} \mathrm{5,}$ and 6).

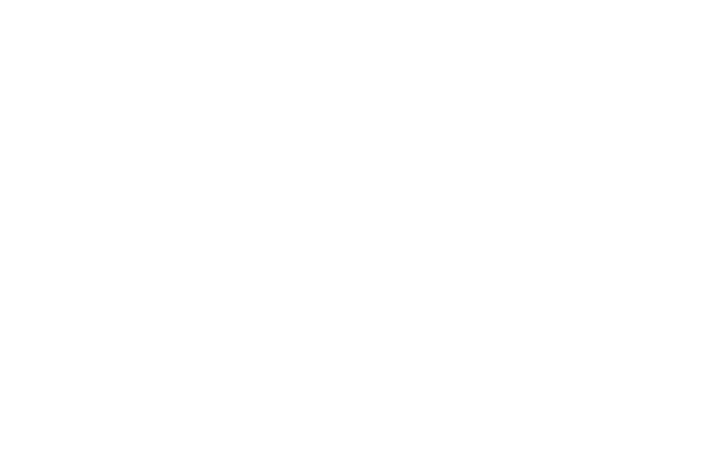

Fig. 4. Effect of testosterone (T), Dihydrotestosterone (DHT), and $17 \beta$-Estradiol (E).

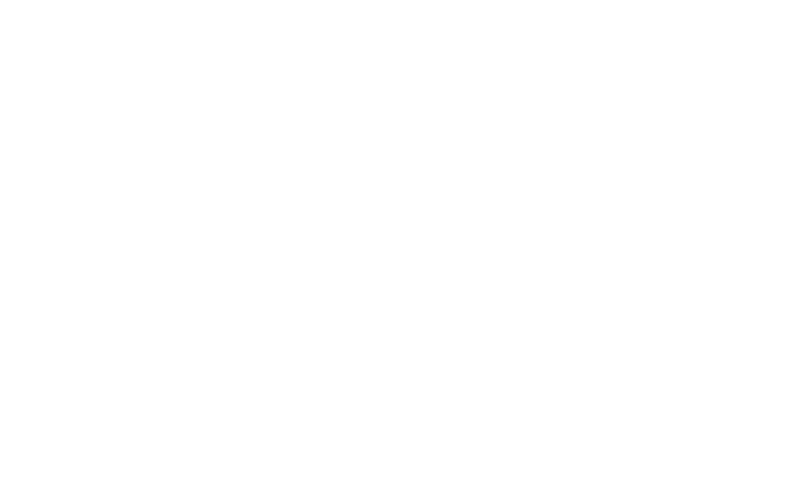

Fig. 5. Effect of testosterone (T), Dihydrotestosterone (DHT), and $17 \beta$-Estradiol (E) on Adipo Q mRNA.

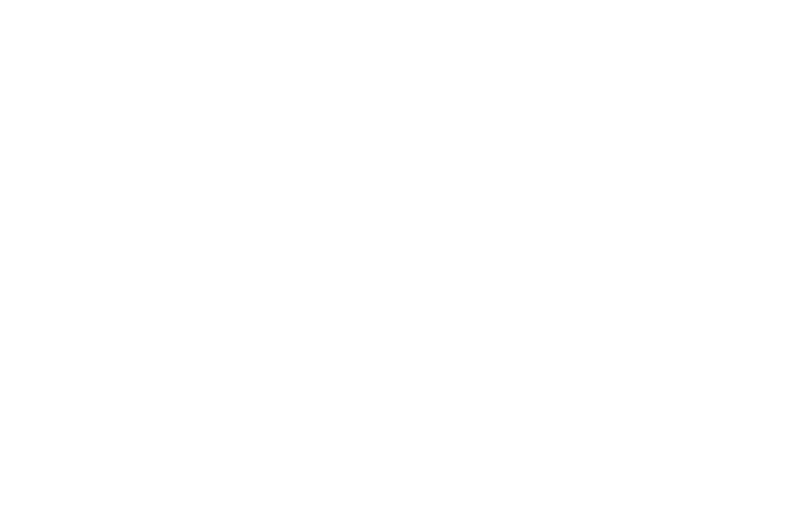

Fig. 6. Effect of testosterone (T), Dihydrotestosterone (DHT), and 17 $\beta$-Estradiol (E) on FABP 4 mRNA.

\section{Discussion}

Adipocytes are endocrine cells for energy storing. Their differentiation is an important process for maintaining physiological cellular function [23], [24]. Inhibition of adipocyte differentiation offers an important therapeutic approach for the prevention and treatment of obesity. There is a need to consider the role of environmental and genetic factors in incidence of chronic diseases. In this study, we have tried to obtain initial results to establish scientific plans for future studies.

The aim of this paper was to investigate the dose-dependent effects of different streoid hormones on adipogenesis in 3T3-L1 cells, by comparing the accumulation of fat between different doses of Testosterone, DHT and Estradiol. Inhibition of adipocyte differentiation offers an important therapeutic approach for the prevention and treatment of obesity. The reasons for this chapter are that we have tried to obtain initial results to establish scientific plans for future studies.

Using Oil Red 0 staining clearly demonstrated that differentiation of 3T3-L1 is observed on day 15 with obvious lipid droplets. This step is important to have evidence about the 3T3-L1 pre-adipocytes' ability to 
accumulate fat. There are a few data being available to explain effect of sex steroids on adipogenic markers at later stage of adipogenic differentiation.

In a series of studies, Singh and colleagues (2006) demonstrated that Testosterone (10, 30, and $100 \mathrm{nM})$ and DHT $(1,3,10 \mathrm{nM}$ ) for 12 days starting at day 2 (after induction) inhibited the differentiation of 3T3-L1 pre-adipocytes into mature adipocytes and down-regulated the mRNA and protein expression of key adipogenic transcription factors for early stages of differentiation, such as C/EBP alpha, C/EBP beta and PPAR gamma 2, in a time and concentration-dependent manner [25]. In contrast, we observed that a nonsignificant decrease in adipogenic markers (resistin, and FABP 4) in 3T3-L1 cells treated with sex steroid (5, 15, $30 \mathrm{nM}$ ) for 13 days, but there were no changes in Adipo Q mRNA expression. These data support the need to conduct more results to investigate the effect of sex steroid at different doses with time courses especially during early stage of differentiation, on transcription factors such as PPAR gamma 2, CEBP alpha, and CEBP betta.

\section{Acknowledgments}

This work was supported by King Saud University, Saudi Arabia and conducted by Nutritional Science division, the university of Nottingham, UK.

\section{References}

[1] Wang, Y. C., Colditz, G. A., \& Kuntz, K. M. (2007). Forecasting the obesity epidemic in the aging US population. Obesity, 15(11), 2855-2865.

[2] Deitel, M. (2003). Overweight and obesity worldwide now estimated to involve 1.7 billion people. Obesity Surgery, 13(3), 329-330.

[3] Finkelstein, E. A., et al. (2012). Obesity and severe obesity forecasts through 2030. Am. J. Prev. Med., 42(6), 563-570.

[4] Gesta, S., Tseng, Y. H., \& Kahn, C. R. (2007). Developmental origin of fat: Tracking obesity to its source. Cell, 131(2), 242-256.

[5] Tuomilehto, J., Schwarz, P., \& Lindstrom, J. (2011). Long-term benefits from lifestyle interventions for type 2 diabetes prevention: Time to expand the efforts. Diabetes Care, 34 Suppl 2, 210-214.

[6] World Health Organization (2011). Global Status Report on Noncommunicable Diseases 2010. Geneva, Switzerland: World Health Organization.

[7] Finkelstein, E. A., et al. (2009). Annual medical spending attributable to obesity: Payer-and servicespecific estimates. Health Aff (Millwood), 28(5), 822-831.

[8] Jones, A. G., et al. (2013). Practical implications of choice of test in National Institute for Health and Clinical Excellence (NICE) guidance for the prevention of Type 2 diabetes. Diabet. Med., 30(1), 126-127.

[9] Sethi, J. K., \& Vidal-Puig, A. J. (2007). Targeting fat to prevent diabetes. Cell Metabolism, 5(5), 323-325.

[10] Gregoire, F. M. (2001). Adipocyte differentiation: From fibroblast to endocrine cell. Experimental Biology and Medicine, 226(11), 997-1002.

[11] Mauriege, P., et al. (1993). Abdominal and femoral adipose-tissue lipolysis and cardiovascular-disease risk-factors in men. European Journal of Clinical Investigation, 23(11), 729-740.

[12] Abate, N., \& Garg, A. (1995). Heterogeneity in adipose tissue metabolism: causes, implications and management of regional adiposity. Prog. Lipid Res., 34(1), 53-70.

[13] Ross, S. E., et al. (2000). Inhibition of adipogenesis by Wnt signaling. Science, 289(5481), 950-953.

[14] Cooke, P. S., \& Naaz, A. (2004). Role of estrogens in adipocyte development and function. Experimental Biology and Medicine, 229(11), 1127-1135.

[15] Ness-Abramof, R., \& Apovian, C. M. (2008). Waist circumference measurement in clinical practice. 
Nutrition in Clinical Practice, 23(4), 397-404.

[16] Yamamoto, S., et al. (2010). Visceral fat area and markers of insulin resistance in relation to colorectal neoplasia. Diabetes Care, 33(1), 184-189.

[17] Palou, M., et al. (2009). Gene expression patterns in visceral and subcutaneous adipose depots in rats are linked to their morphologic features. Cellular Physiology and Biochemistry, 24(5-6), 547-556.

[18] Rodriguez, E., et al. (2004). PPAR-gamma 2 expression in response to cafeteria diet: Gender- and depotspecific effects. Obes. Res., 12(9), 1455-1463.

[19] Gapstur, S. M., et al. (2002). Serum androgen concentrations in young men: A longitudinal analysis of associations with age, obesity, and race. The CARDIA male hormone study. Cancer Epidemiology Biomarkers and Prevention, 11(10 Part 1), 1041-1047.

[20] Arias-Santiago, S., et al. (2011). Sex hormone-binding globulin and risk of hyperglycemia in patients with androgenetic alopecia. J. Am. Acad. Dermatol., 65(1), 48-53.

[21] Allan, C. A., et al. (2008). Testosterone therapy prevents gain in visceral adipose tissue and loss of skeletal muscle in nonobese aging men. Journal of Clinical Endocrinology \& Metabolism, 93(1), 139-146.

[22] Ye, Z. W., Wu, X. M., \& Jiang, J. G. (2010). Knockdown of angiotensinogen by shRNA-mediated RNA interference inhibits human visceral preadipocytes differentiation. International Journal of Obesity, 34(1), 157-164.

[23] Feve, B. (2005). Adipogenesis: Cellular and molecular aspects. Best Pract. Res. Clin. Endocrinol. Metab., 19(4), 483-499.

[24] Ali, A. T., et al. (2013). Adipocyte and adipogenesis. Eur. J. Cell Biol., 92(6-7), 229-236.

[25] Singh, R., et al. (2006). Testosterone inhibits adipogenic differentiation in 3T3-L1 cells: Nuclear translocation of androgen receptor complex with beta-catenin and T-cell factor 4 may bypass canonical Wnt signaling to down-regulate adipogenic transcription factors. Endocrinology, 147(1), 141-154.

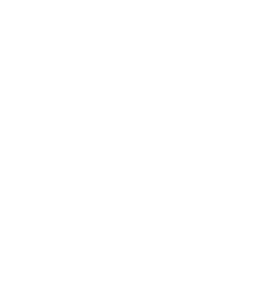

Ghedier Alshammari received his PhD degree in molecular nutrition, from University of Nottingham, He is currently a lecturer in the Department of Food Sciences and Nutrition, College of Food and Agricultural Sciences, King Saud University, Riyadh, Saudi Arabia. 